\title{
Turbulent helium gas cell as a new paradigm of daily meteorological fluctuations?
}

\author{
I.M. Jánosi ${ }^{1}$, G. Vattay ${ }^{2}$ and A. Harnos ${ }^{2}$
}

October 25, 2018

\author{
${ }^{1}$ Physics of Complex Systems Group, Eötvös University, H-1088 Budapest, \\ Múzeum krt. 6-8, Hungary \\ ${ }^{2}$ University of Veterinary Science, Department of Biomathematics and \\ Computer Science, István u. 2, H-1078 Budapest, Hungary
}




\begin{abstract}
We compare the spectral properties of long meteorological temperature records with laboratory measurements in small convection cells. Surprisingly, the atmospheric boundary layer sampled on a daily scale shares the statistical properties of temperature fluctuations in small-scale experiments. This fact can be explained by the hydrodynamical similarity between these seemingly very different systems. The results suggest that the dynamics of daily temperature fluctuations is determined by the soft turbulent state of the atmospheric boundary layer in continental climate.
\end{abstract}

The studies of turbulence in the atmospheric boundary-layer has a history of almost hundred years. The theoretical description of the basic mechanisms has been elaborated in detail [1], however the application of the concept of turbulence at the level of observed data evaluation is still not complete. For example, one of the most common meteorological quantities measured for a long time at various frequencies is the temperature. The length and the relative accuracy of the available time series make these records very attractive for detailed statistical analysis. Indeed, there have been several attempts to describe the dynamics of daily medium temperature fluctuations. A typical viewpoint is that the underlying mechanism is fully stochastic in nature and can be considered as an autoregressive process [2]. A completely different viewpoint suggests that the apparent irregularities may be attributed to a deterministic chaotic behavior, although serious doubts have arisen on the existence of low dimensional chaos in the long time behavior of the atmosphere (climatic attractor) [3], as well as in the processes over short time scales 任.

Our study based on daily temperature measurements of the Hungarian Meteorological Service performed at twenty different meteorological stations covering the area of Hungary for the period 1951 - 1989. We compare the statistical properties of our time series with measurements on local temperature fluctuations in gaseous helium and air [6, 7, 8] performed by the Chicago Group. A typical experimental setup for studying free thermal convection consists of a closed container filled with different gases or liquids (Fig. 1), and the local temperature inside is recorded on a millisecond scale.

Let us start with the characterization of the meteorological data. These records show a systematic seasonal periodicity and superimposed daily random fluctuations, a detailed analysis has been published in Ref. [5]. We produced the fluctuation time series by subtracting the daily average over 39 years from the daily medium temperature data. Thus, the fluctuation $T_{f}(d, y)$ on day $d$ of year $y$ is given by the deviation of the actual daily medium $T(d, y)$ from the seasonal average $\langle T\rangle(d)$, i.e.:

$$
T_{f}(d, y)=T(d, y)-\langle T\rangle(d)
$$


where $\langle T\rangle(d)$ is defined by

$$
\langle T\rangle(d)=\frac{1}{39} \sum_{y=1951}^{1989} T(d, y) .
$$

The histogram of the fluctuation amplitudes has a typical Gaussian distribution (Fig. 2). The power density spectrum of the time series measured at different meteorological stations can be fitted well by the function (see Fig. 3):

$$
P(f)=P_{s} \exp \left[-\left(\frac{f}{f_{s}}\right)^{\beta}\right],
$$

where $P_{s}=222 \pm 16, f_{s}=0.017 \pm 0.005$ day $^{-1}, \beta=0.54 \pm 0.03$, here the deviations indicate slight meteorological station dependence. Note that the end of the spectra could be approximated with a power-law shape as well, but only on a narrow frequency range (less than a half order of magnitude). Surprisingly, the fitting form Eq. (1) with the same exponent $\beta$ and the Gaussian fluctuation histogram are the same as was found in experiments on convecting helium [6, 7], in the so-called soft turbulent regime. How can one relate atmospheric phenomena to small-scale model experiments?

It is well known that two hydrodynamical systems are similar, if their dimensionless parameters are equal. In the case of experiments on thermal convections, the analysis is usually based upon the Boussinesq approximations [1], which give two dimensionless parameters. First, the Rayleigh number is given by

$$
\mathrm{Ra}=\frac{\alpha g L^{3} \Delta T}{\kappa \nu},
$$

where $\alpha$ is the (isobar) thermal expansion coefficient, $g$ is the gravitational acceleration, $L$ is the height of the container, $\Delta T=T^{+}-T^{-}$is the temperature difference between the top and bottom plates (Fig. 1), $\kappa$ is the thermal diffusivity, while $\nu$ is the kinematic viscosity. Second, the Prandtl number is simply the ratio

$$
\operatorname{Pr}=\frac{\nu}{\kappa} .
$$

These two numbers together with the aspect-ratio of the cell $(A=$ width/height $)$ give a complete description of the experimental system.

The material parameters of the air do not depend drastically on the meteorological circumstances, some typical values are the following [1, 10]: $\alpha=$ $3.55 \times 10^{-3} 1 / \mathrm{K}, g=9.81 \mathrm{~m} / \mathrm{s}^{2}, \kappa=2.03 \times 10^{-5} \mathrm{~m} / \mathrm{s}^{2}, \nu=1.44 \times 10^{-5} \mathrm{~m} / \mathrm{s}^{2}$. Between 0 and $40{ }^{\circ} \mathrm{C}$, the Prandtl number remains constans: $\operatorname{Pr}^{\text {air }}=0.71$. The Prandtl number of the helium gas was kept also on a constant value $\operatorname{Pr}^{\mathrm{He}}=0.64$ in the experiments [6, 7].

Three important points have to be considered for the proper comparison of the observations, because the atmospheric boundary layer differs in some 
respects from the laboratory setups. First, there is no well-defined top plate above the gas layer. This makes the term $L^{3} \Delta T$ in Eq. (2) indefinite, therefore the direct determination of an atmospheric Rayleigh number is impossible. Fortunately, there are two characteristic frequencies in the power spectra, and a proper matching (see later) can provide an alternative estimation for the length $L$. Second, the atmospheric boundary layer has practically an infinite aspect ratio, while the laboratory experiments were performed in cells of low aspect ratios. This problem can be resolved by observations, which suggest that the vertical and horizontal sizes of the medium scale $(\sim 100-500 \mathrm{~m})$ convective eddies are approximately equal. Third, adequate measurements show that a characteristic turnover time of these eddies is in the range of minutes, while the sampling rate of the meteorological records is one day. Note, however, that the meteorological time series consist of daily medium temperature data, that is the mean of the daily absolute maximum and minimum values. Indeed, this can differ from the daily average temperature, and may reflect of effects on much shorter time scale than one day.

The control parameter in the laboratory experiments is the Rayleigh number Ra. The Chicago Group covered the range of Ra from $10^{5}$ to $10^{11}$ by changing the pressure of different gases [6, 7, 8]. After the onset of convection, two different chaotic domains $\mathrm{Ra}=1.5-2.5 \times 10^{5}$ and $\mathrm{Ra}=2.5-5 \times 10^{5}$ with attractor dimensions $D \approx 2$ and $D \approx 4$ were identified. There are two regimes after the onset of turbulence: The soft turbulent state $\left(5 \times 10^{5}<\mathrm{Ra}<\mathrm{Ra}_{c}\right)$ and hard turbulent state $\left(\mathrm{Ra}>\mathrm{Ra}_{c}\right)$, where $\mathrm{Ra}_{c} \approx 10^{8}$ depending slightly on the aspect ratio $A$. The probability distribution of the local temperature fluctuations is Gaussian in soft turbulence, while exponential in hard turbulence. The power spectrum of the fluctuations is stretched-exponential in soft turbulence [Eq. (1)], while it is a power-law with an exponent $-7 / 5$ in hard turbulence. The Gaussian amplitude distribution (Fig. 2) and the stretched-exponential power spectrum (Fig. 3) of the meteorological time series suggest that the atmospheric boundary layer may exhibit soft turbulence, let us analyse further this assumption.

It is known from the experiments [6, 月, 8], how the characteristic frequencies of the power spectra depend on Ra. First, the fitted frequency $f_{s}$ of Eq. (1) (measured in units of $\kappa / L^{2}$ ) can be estimated from Fig. 3 of Ref. [6] in the soft turbulent regime as $f_{s}(\mathrm{Ra}) \approx 63 \kappa / L^{2}$ for $\mathrm{Ra} \approx 10^{6}$, and $f_{s}(\mathrm{Ra}) \approx 13000 \kappa / L^{2}$ for $\mathrm{Ra} \approx 10^{8}$. Another characteristic value is the cutoff-frequency $f_{\text {max }}$, at which the power spectra flatten out as a consequence of noise (Fig. 4). This frequency depends also on Ra, moreover the relationship does not change at the transition of soft-hard turbulence. From Fig. 13 of Ref. [8], one can estimate $f_{\max }(\mathrm{Ra}) \approx 2500 \kappa / L^{2}$ for $\mathrm{Ra} \approx 10^{6}$, and $f_{\max }(\mathrm{Ra}) \approx 10^{5} \kappa / L^{2}$ for $\mathrm{Ra} \approx 10^{8}$. The values for $f_{\max }$ depend on the distance of thermistor from the fix plate [8], but not to the extent that it could affect our following order of magnitude estimation. Both the characteristic frequency $f_{s}=2 \times 10^{-7} \mathrm{~Hz}$ of Eq. (1) and the the cutoff frequency $f_{\max }=4.5 \times 10^{-6} \mathrm{~Hz}$ of Fig. 4, yield to an estimation $L \approx 80-1100 \mathrm{~m}$ in the atmospheric boundary layer. Note that this seemingly 
wide interval belongs to a Rayleigh number range of $10^{6}<\mathrm{Ra} 10^{8}$. On the other hand, this height $L$ is in agreement with the accepted values of the thickness of the air layer influenced by the daily cycle of temperature change [11].

These results indicate that the typical atmospheric dynamics will unlikely exhibit low dimensional chaos, since soft turbulent state occurs after several transitions from the chaotic state and is connected with the increase of effective degrees of freedom. Furthermore, the present concept makes a strong prediction on daily temperature fluctuations at extreme climatical conditions. In such a case, the Rayleigh number can exceed the threshold value $\mathrm{Ra}_{c}$, and then hard turbulence develops. Indeed, recent studies on North Pole data 12] suggest, that characteristics of hard turbulence can be observed.

Finally, we would like to mention, that daily fluctuations of other meteorological data often show non-trivial scaling behaviour. For example, relative air humidity fluctuations have $1 / f^{\alpha}(\alpha \approx 0.61)$ power spectrum [13, for more than two decades of frequency range. Power-law scaling of frequency spectra is the typical sign of nontrivial complex behaviour and self-organization. This implicitly confirms the turbulence based explanation of fluctuations on a daily scale, since density fluctuations, like those of air humidity, in turbulent flows, are expected to have power-law frequency scaling. It would be interesting to analyze the daily fluctuations measured at different meteorological conditions, world wide, from the point of view outlined here. By doing this, we could learn how different climates affect the transition between hard and soft turbulence and draw conclusions how global warming, ice ages, natural catastrophes and other large scale climate changes affect the distribution of temperature measured on the ground. This information could be useful, since a transition toward hard turbulence increases the occurrence of large daily fluctuations of order $\pm 20^{\circ} \mathrm{C}$. A shift toward a power law frequency spectra increases the occurrence of extremely long correlated time periods when the temperature significantly deviates from the seasonal average values.

This work has been supported by the "Nonlinear Phenomena" EU Research Network and the Hungarian National Science Foundation (OTKA).

\section{References}

[1] J. R. Garratt, The atmospheric boundary layer (Cambridge University Press, Cambridge, 1992).

[2] P. Talkner, R. Weber, and W. Roser, in Annual Report of Paul Scherrer Institut (PSI, Villigen, 1990) p. 78-84.

[3] B. Legras, Phys. World 3, 29-32 (1990).

[4] T. Bountis, L. Karakatsanis, G. Papaioannou, and G. Pavlos, Ann. Geophysicae 11, 947-959 (1993). 
[5] I. M. Jánosi and G. Vattay, Phys. Rev. A 46, 6386-6389 (1992); in Noise in Physical Systems and $1 / f$ Fluctuations edited by P.H. Handel and A. L. Chung, AIP Conf. Proc. No. 285, (AIP Press, New York, 1993), p. 574-577.

[6] F. Heslot, B. Casting and A. Libchaber, Phys. Rev. A 36, 5870-5873 (1987).

[7] X. Z. Wu, L. Kadanoff, A. Libchaber and M. Sano, Phys. Rev. Lett. 64, 2140-2143 (1990).

[8] A. Belmonte, A. Tilgner, and A. Libchaber, Phys. Rev. E 50, 269-279 (1994)

[9] W. H. Press, S. A. Teukolsky, W. T. Vetterling, and B. P. Flannery, Numerical Recipes, 2nd ed. (Cambridge University Press, Cambridge, 1992).

[10] V. V. Sychev et al., Thermodynamic properties of air, (Hemisphere Publishing Corp., New York, 1987).

[11] J. R. Holton, An Introduction to Dynamical Meteorology, (Academic, New York, 1972)

[12] H. Svensmark, N. W. Nielsen, A. M. Sempreviva, Large Scale Soft and Hard Turbulent States of the Atmosphere preprint, Danish Meteorological Institute (1995)

[13] G. Vattay and A. Harnos, Phys. Rev. Lett. 73, 768-771 (1994). 


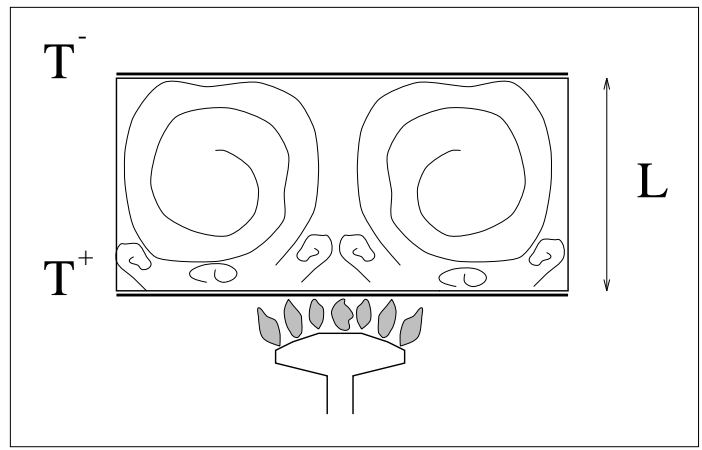

Figure 1: Scematic of typical experimental setups for studying free thermal convection. A characteristic size of the gas container is usually $L=10-40 \mathrm{~cm}$. The bottom plate is heated $\left(T^{+}\right)$, while the top plate is kept on a constants $T^{-}$ (lower) temperature. The instantaneous gas temperature inside is measured by small thermistors placed to a desired location. The atmospheric boundary layer bears a close resemblance to this experiment: The air above the ground surface is heated also from below and cooled at the top by higher air layers. 


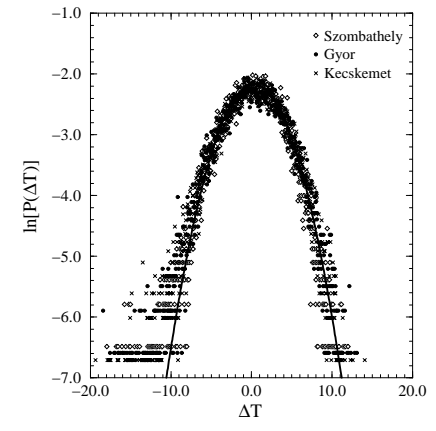

Figure 2: Normalized probability distributions for the detrended daily medium temperature fluctuations measured between 1.1.1951-31.12.1989 in three different stations. The solid line shows a Gaussian fit with a center of $0.31{ }^{\circ} \mathrm{C}$ and standard deviation of $3.50{ }^{\circ} \mathrm{C}$. The Gaussian shape together with the slight deviation at the left side (note the logarithmic scale) is the same for all of the twenty evaluated records [5]. 


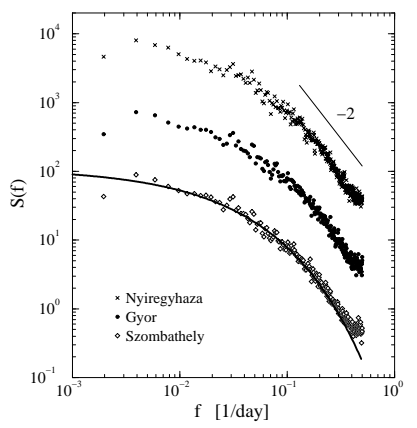

Figure 3: Unnormalized power density spectrum of the temperature fluctuations measured in three stations. The frequency unit is day ${ }^{-1}$. The upper two spectra are shifted upwards for the sake of clearness. The result were obtained by a standard Fast Fourier Transformation method using Hann windowing [9]. The thick solid line is the fit given by Eq. (1), the thin line illustrates an $1 / f^{2}$ behavior on a restricted frequency range. 


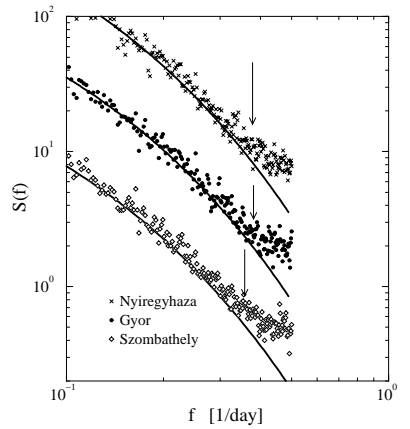

Figure 4: The same as Fig. 3 zoomed to the end of the spectra. The arrows indicate the cutoff frequencies $f_{\max }=0.39 \pm 0.02 \mathrm{day}^{-1}$, where the spectra reaches the noise level. 